\title{
Sexuelle Übergriffe durch Medizinalpersonen
}

W. Tschan

Seit Oktober 2001 ist die von der Medizinischen Gesellschaft Basel geschaffene PABS (PatientenAnlauf- und -Beratungsstelle) nun operativ im Einsatz [1]. Sechs Ärztinnen und Ärzte aus unterschiedlichen fachlichen Bereichen (Allgemeinmedizin, Innere Medizin, Gynäkologie, Psychiatrie) nehmen die primäre Beratungsaufgabe von Patienten und Angehörigen wahr, welche sexuelle Übergriffe durch Fachleute im Gesundheitswesen erlebt haben. In Zusammenarbeit mit der Medizinischen Notrufzentrale wird eine 24-Stunden-Hotline betrieben, wo sich Betroffene und Ratsuchende jederzeit melden können.

\section{PABS-Hotline 0615601550}

Eine dreiköpfige Steuerungsgruppe ist für den Betrieb der PABS verantwortlich. Zu ihren Aufgaben gehört neben der Klärung von Schnittstellenfragen die Sicherstellung einer spezifischen Ausbildung der beteiligten Ärztinnen und Ärzte. Mangels geeigneter Angebote konzipierte die Steuerungsgruppe eine modulartige Ausbildung, welche 2001/02 ein erstes Mal durchgeführt wurde und die erforderliche Faktenkenntnis vermittelte. Bereits im Rahmen der ersten Durchführung ermöglichte die Medizinische Gesellschaft die Teilnahme für interessierte Kolleginnen und Kollegen.

\section{Interdisziplinäre Wissensvermittlung}

Die Problematik von PSM (Professional Sexual Misconduct) wirft eine Vielzahl von Fragestellungen auf. Kenntnisse aus unterschiedlichen Bereichen sind erforderlich, um in dieser schwierigen Materie eine kompetente Beratung anbieten zu können. PSM ist nicht bloss eine rechtliche Thematik, sondern erfordert beispielsweise auch Kenntnisse über die Behandlungsmöglichkeit betroffener Personen. Forensische Kenntnisse über Sexualstraftäter sind ebenso Voraussetzung wie auch Wissen über Epidemiologie, Ursachen und Pathophysiologie von PSM. Der «single disciplinary effect» [2] der traditionellen Ausbildung muss durch eine interdisziplinäre Wissensvermittlung überwunden werden. Durch die Auswahl der Referentinnen und Referenten wie auch der Themenschwerpunkte wurde diesen Überlegungen Rechnung getragen. Den Teilnehmern wurde Gelegenheit geboten, aus erster Hand die unterschiedlichen Perspektiven der involvierten Bereiche kennenzulernen. Eindrücklich war die Falldarstellung einer Strafgerichtspräsidentin, welche über die in der Schweiz erstmalige Verurteilung eines Arztes wegen Ausnutzung der Abhängigkeit nach Art. 193 StGB berichtete. Das Urteil war wenige Tage zuvor gefällt worden.

\section{Der neue Ausbildungszyklus}

Die Medizinische Gesellschaft Basel hatte festgelegt, dass die PABS-Ärztinnen und -Ärzte eine regelmässige Weiterbildung in dieser spezifischen Thematik absolvieren können. Damit stellte sich für die Steuerungsgruppe erneut die Aufgabe, ein Weiterbildungsangebot zu konzipieren. Wiederum konnten zahlreiche renommierte Fachleute für diese Aufgabe gewonnen werden. Die WHO zeigte sich am Pilotvorhaben interessiert und wird durch einen Beitrag die Problematik für das Gesundheitswesen aufzeigen. Für die WHO stellen die Thematisierungen von PSM eine sinnvolle Ergänzung zur laufenden Kampagne über die Prävention von sexueller Gewalt und die Auswirkungen auf das Gesundheitswesen dar [3]. Nationale und kantonale Behörden, Experten aus Justiz, Forensik, Psychotraumatologie und Qualitätssicherung haben ihre Teilnahme zugesagt. Der Ausbildungszyklus bietet für alle interessierten Kolleginnen und Kollegen eine hervorragende Möglichkeit, sich mit der komplexen Materie vertraut zu machen. Im Rahmen eines Einführungsseminars werden ein Überblick über den Stand des Wissens sowie aus der praktischen Erfahrung gewonnene Interventionsstrategien vermittelt. Die Veranstaltung ist von besonderem Interesse für alle in der Grund- und Notfallversorgung tätigen Fachärzte, insbesondere aus der Pädiatrie, Gynäkologie, Allgemein- und Inneren Medizin sowie Psychiatrie. Ohne Kenntnisse der Pathophysiologie sind weder Diagnostik noch Behandlung möglich. 


\section{«Reporting» als Voraussetzung weiterer Massnahmen}

Wir haben in letzter Zeit wiederholt über die Notwendigkeit eines «critical incident reporting» [4] als Voraussetzung einer effektiven Qualitätssicherung gehört. Das «Reporting» gilt im Bereich von Präventionsmassnahmen als wesentlichste Massnahme - ohne Kenntnis des Ausmasses und der Auswirkungen wird kein Handlungsbedarf gesehen. Welche Strategien sich anschliessen müssen - etwa Rehabilitationsprogramme für betroffene Fachleute im Sinne von Hilfe satt Strafe [5] - ist Gegenstand aktueller Diskussionen [6]. Eine Arbeitsgruppe der FMH wird mögliche Vorgehensweisen aufzeigen. Der Ausbildungszyklus wird mögliche Strategien und Lösungsansätze aufzeigen.

\section{Zusammenfassung}

- Die einzelnen Massnahmen in Zusammenhang mit sexuellen Übergriffen durch Medizinalpersonen können nicht isoliert betrachtet werden.

- Der Wissensvermittlung zu diesen Fragen kommt eine entscheidende Bedeutung zu.

- Ein interdisziplinärer Zugang ist angesichts der Vielschichtigkeit der Problematik notwendig.

\section{Hinweis}

Programm und Online-Anmeldung auf der Homepage der Medizinischen Gesellschaft Basel: www.medges.ch $\rightarrow$ Patientenberatung $\rightarrow$ PABS Ausbildung 2004/2005

\section{Literatur}

1 Tschan W. PABS: Patienten-Anlauf- und -Beratungsstelle der Medizinischen Gesellschaft Basel. Schweiz Ärztezeitung 2001;82(47):2467-73.

2 Fegert JM, Berger C, Klopfer U, Lehmkuhl U, Lehmkuhl G. Umgang mit sexuellem Missbrauch. Institutionelle und individuelle Reaktion. Münster: Votum Verlag; 2001.

3 Krug EG, Dahlberg LL, Mercy JA, Zwi AB, Lozano R. World report on violence and health. Geneva: World Health Organisation; 2002.

4 Kaufmann M, Scheidegger D. Anonymes Critical Incident Reporting: Ein Beitrag zur Patientensicherheit (Lernen aus Fehlern). Synapse 2004; 2:1-4.

5 Tschan W. Umgang mit Tätern. Schweiz Ärztezeitung 2003;84(30):1568-9.

6 Tschan W. Missbrauchtes Vertrauen. Grenzverletzungen in professionellen Beziehungen. Basel: Karger; 2001. 